\title{
Some Beam-Loading Problems in LAMPF II
}

\author{
Tai-Sen F. Wang \\ John L. Warren
}

\section{DISCLAIMER}

This report was prepared as an account of work sponsored by an agency of the United States Government. Neither the United States Government nor any agency thereof, nor any of their employees, makes any warranty, express or implied, or assumes any legal liability or responsibility for the accuracy, completeness, or usefulness of any information, apparatus, product, or process disclosed, or represents that its use would not infringe privately owned rights. Reference herein to any specific commercial product, process, or service by trade name, trademark, manufacturer, of otherwise does not necessarily constitute or imply its endorsement, recommendation, or favoring by the United States Government or any agency thereof. The views and opinions of authors expressed herein do not necessarily state or reflect those of the United States Government or any agency thereof.
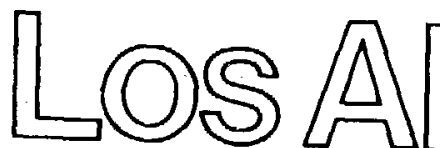


\title{
SOME BEAM-LOADING PROBLEMS IN LAMPF II
}

by

Tai-Sen F. Wang and John L. Warren

\begin{abstract}
Some beam-loading problems are investigated for the proposed LAMPF II synchrotron main ring and booster. Estimations for the feedback control requirements to reduce the power consumption, by accelerating protons beyond the Robinson stability limit, are provided. The results indicate that it is technically feasible to realize this kind of power-saving idea.
\end{abstract}

\section{INTRODUCTION}

The current design of LAMPF II calls for two synchrotrons: a rapid-cycling $60-\mathrm{Hz}, 9-\mathrm{GeV}$ booster that feeds a $6-\mathrm{Hz}, 45-\mathrm{GeV}$ main ring. The booster will deliver 2 pulses in 10 to the main ring and the other 8 pulses to a neutrino production facility. The booster may also operate in a $30-\mathrm{Hz}, 12-\mathrm{GeV}$ mode when it is not supplying beam to the main ring. Figure 1 schematically shows the time-dependence $c$ ? average circulating beam current.

Actually, the circulating beam in the booster consists of 120 bunches containing up to $1.73 \times 10^{11}$ protons with the head and tail of the bunch train separated by 12 empty rf buckets (harmonic number $h=132$ ). The main ring contains 240 bunches with two 12-bucket gans. The main ring is filled in two booster pulses, each with about 2.2-us injection time. For about $16.7 \mathrm{~ms}$, the main ring is only half filled. We will show below that the empty buckets have a minor effect on the rf-system, but the transient caused by the halffilled main ring will require either fast feedback on the rf-power supply or some feedforward control.

The main concern of this report is the stabilization of the synchrotron motion of the beam by means of feedback in the face of the Robinson instability caused by heavy beam loading of the rf-cavities.' Griffin ${ }^{2}$ has shown that feedback stabilization will work, but great care must be taken in the design of the feedback system. Griffin has also shown that the Robinson stability 

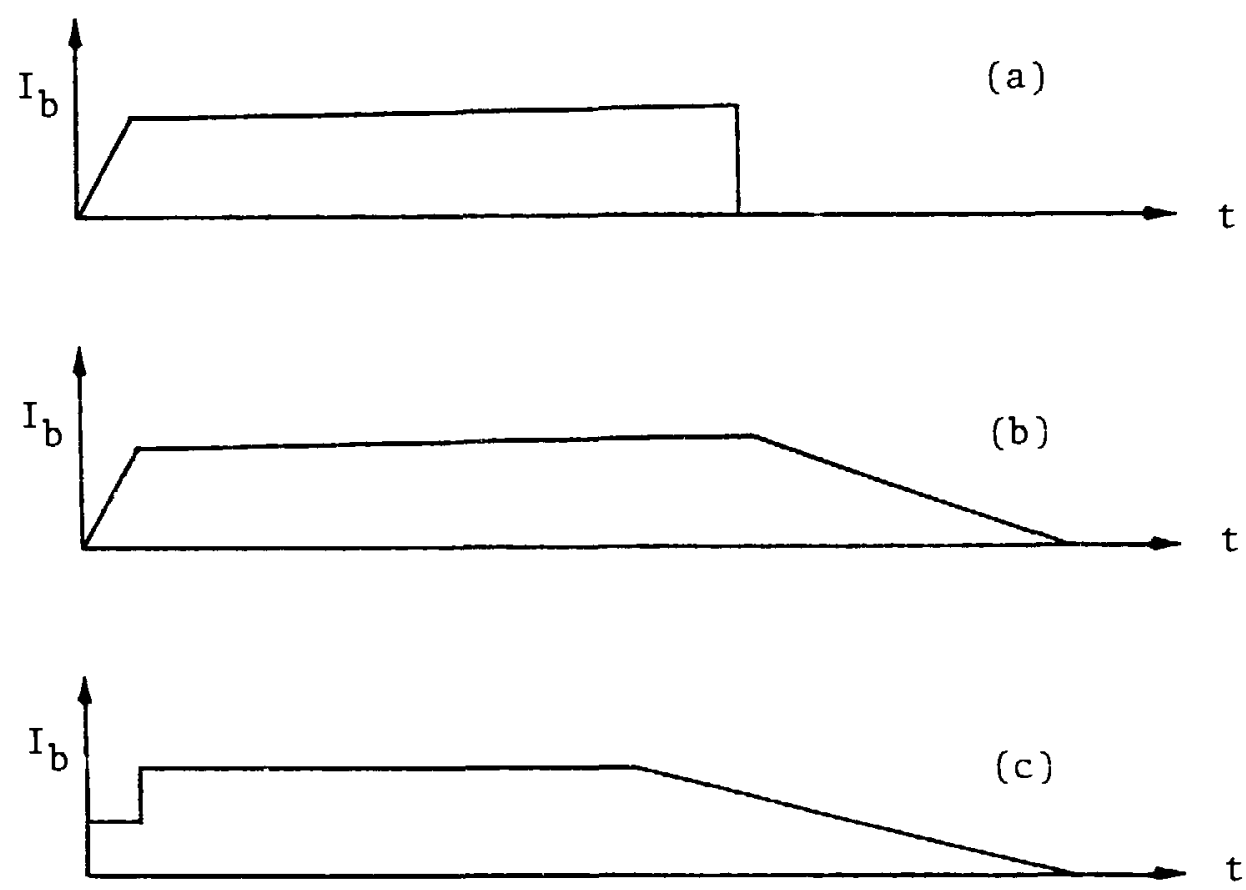

Fig. 1. Schematic drawing of the time dependence of average beam current for (a) the $60-\mathrm{Hz}, 9-\mathrm{GeV}$ booster with fast extraction, (b) the $30-\mathrm{Hz}$, 12-GeV booster with slow extraction, and (c) the 6-Hz, 45-GeV main ring with slow extraction.

criterion is equivalent to requiring that the ratio $P_{R}$ of power delivered to the beam to power dissipated in the cavities be less than one. ${ }^{2}$ For reasons of efficiency and ecoriomics, it is desired to operate with $P_{R} \geq 1$. This requires feedback stahilization.

We have analyzed the LAMPF II synchrotrons using the methods similar to those in Refs. 3-6, but with somewhat different feedback functions. To explain our notation and to make this repcrt relatively self-contained, we have rederived the key equations. In the figures at the end of the report, the final results are shown, along with the input parameters for- LAMPF II and the resulting feedback parameters that give reasonable stabilization. The feedback system has not been optimized.

Section II describes the beam-cavity interaction. Section III treats the injection and slow extraction part of the cycle when the rf cavities are acting simply as bunchers. The transient effects from gaps in the beam structure are discussed. Section IV deals with the acceleration phase, the feedback circuit 
models, and an example of the calculational results. Section $V$ summarizes our conclusions.

\section{THEORETICAL MODEL OF BEAM-CAVITY INTERACTION}

The analysis of beam-cavity interaction discussed here will be based on the equivalent circuit model for a cavity driven near resonance. In this model, a cavity is envisioned as a parallel RLC circuit with a tunable inductor; the applied rf power source and the circulating beam current are envisioned as current sources $i_{g}$ and $i_{b}$, respectively. The schematic is shown in Fig. 2.

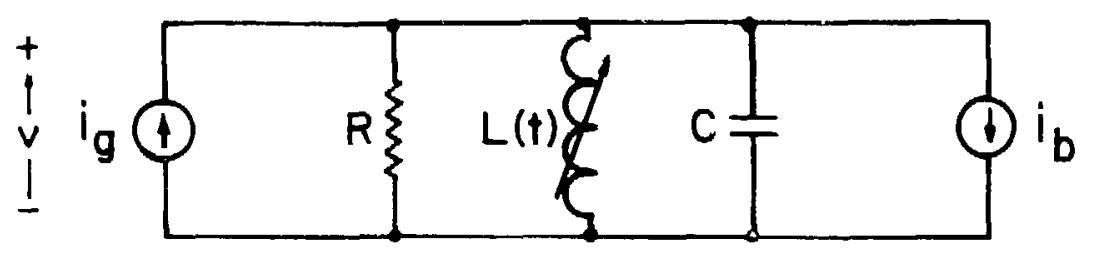

Fig. 2. Equivalent circuit used in analysis.

Using Kirchhoff's law, one can show that the total voltage on the cavity satisfies the differential equation

$\ddot{v}+2 \alpha\left(1-\frac{\dot{\Omega}}{\alpha \Omega}\right) \dot{v}+\Omega^{2}\left(1-\frac{4 \alpha \dot{\Omega}}{\Omega^{3}}\right) v=2 \alpha R\left(\frac{d i_{t}}{d t}-\frac{2 i t^{\prime}}{\Omega}\right)$,

where

$$
\begin{aligned}
L & =L(t)=\text { inductance of the cavity, } \\
C & =\text { capacitance of the cavity, } \\
R & =\text { shunt resistance of the cavity, } \\
\Omega(t) & =1 / \sqrt{L C}=\text { resonant frequency of the cavity, } \\
\alpha & =1 /(2 R C)=\Omega /(2 Q), \\
i_{t} & =i_{g}-i_{b}, \\
Q & =R /(\Omega L)=0 \text { of the cavity, }
\end{aligned}
$$

and the dot indicates the derivative with respect to time $t$. 
For $\Omega \ll \alpha \Omega$, a $\Omega \ll \Omega^{3}$, and $i_{t} \Omega \ll \Omega d i_{t} / d t$, a good approximation to Eq. (1) is

$\ddot{v}+2 \alpha \dot{v}+\Omega^{2} v=2 \alpha R \frac{d i_{t}}{d t}$

More approximations are made by considering only one Fourier component of the beam current near the resonant frequency of the rf cavities. Thus, making the substitution of

$v=\underset{\sim}{v}(t) e^{j \omega t}$,

$i_{g}=\underset{\sim g}{I}(t) e^{j \omega t}$, and

$i_{b}=I_{b}(t) e^{j \omega t}$

in Eq. (2) yields

$\underset{\sim}{\ddot{v}}+2(\alpha+j \omega) \underset{\sim}{\dot{V}}+\left(\Omega^{2}-\omega^{2}+2 j \alpha \omega\right) \underset{\sim}{V}=2 \alpha R(\underset{\sim}{\dot{I}}+j \omega \underset{\sim}{I})$,

where $j^{2}=-1$,

$\omega$ is the frequency of the driving $r f$ power, and

$I=I_{g}-I_{b}$

For synchronization, the $r f$ frequency is chosen according to the relation $\omega=h \omega_{r}$, where $\omega_{r}$ is the revolution frequency of protons and $h$ is the harmonic number. It should be noted here that during acceleration, $\omega$ is actually a function of time. When deriving Eqs. (4) and (5), we have neglected the terms containing $\dot{\omega}$ to avoid complexity. Also, in Eq. (3) we have introduced the 
phasor notation, that is, a symbol above a tilde $(\sim)$ will represent a complex quantity, and the magnitude of a phasor will be represented by the same symbol without the -.

Using the further approximations, $\alpha \ll \omega, \underset{\sim}{\ddot{V}} \ll \omega \underset{\sim}{V}$, and $I \ll \omega I$, Eq. (4) can be further simplified to

$\underset{\sim}{\dot{V}}+\left[\alpha+\frac{j\left(\omega^{2}-\Omega^{2}\right)}{2 \omega}\right]_{\sim}^{v}=\alpha R I$

The relations among these phasors are shown in Fig. 3, where we have chosen a rotating polar coordinate system such that in the steady state, $I_{b}$ should coincide with the real axis. Also in the steady state, we shall use $\psi_{v}$ and $\psi_{g}$ to

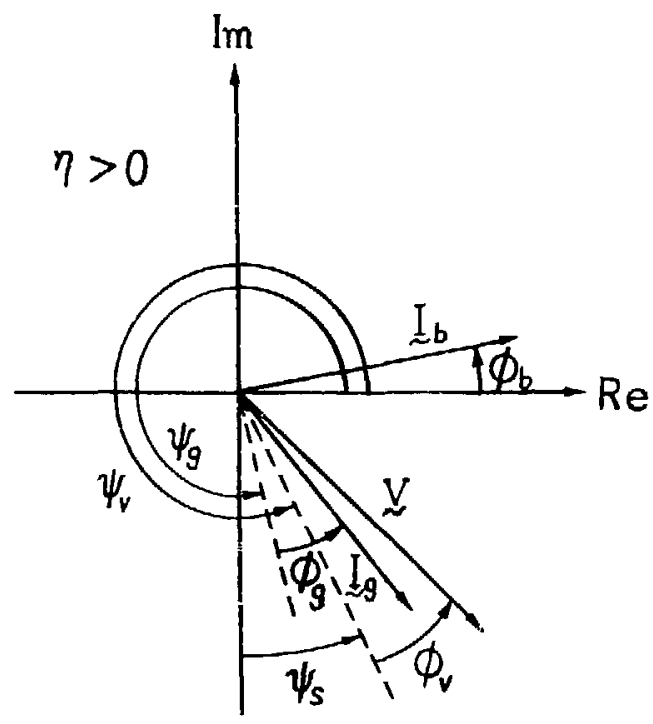

(a)

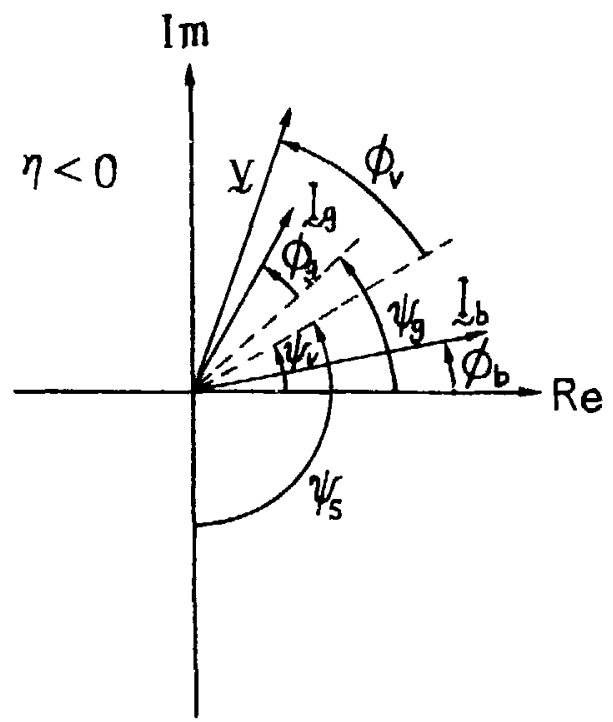

(b)

Fig. 3. Phasor diagram showing the relations among the beam current $I_{b}$, the generator current $I_{g}$, and the total cavity voltage $y$, for (a) below transition, (b) above transition; $\psi_{s}$ represents the synchronous angle and dashed lines represent steady-state angles.

represent the angles of $\underset{\sim}{ }$ and $I_{\sim}$ relative to $I_{b}$, respectively. Systems operating atove $(n<0)$ and below ( $n>0$ ) the transition are separately shown in Fig. 3(a) and Fig. 3(b). The quantity $n$ is defined as 
$n=\frac{1}{r^{2}}-\frac{1}{r_{t}^{2}}$

where $\gamma$ is the relativistic gamma and $\gamma_{t}$ is the transition gamma. For the system under consideration, the phasors will oscillate about their steady states; therefore, we denote $\phi_{v}, \phi_{g}$, and $\phi_{b}$ as the angular deviations of $\underset{\sim}{V}, I_{\sim}$, and $I_{\sim}$ from their steady states. We also introduce the synchronous angle between the total voltage and the beam current $\psi_{s}$, as shown in Fig. 3.

Using the above defined notation, we can write the phasors in polar form as the following:

$$
\begin{aligned}
I_{b} & =I_{b}(t) e^{j \phi_{b}(t)}, \\
I_{g} & =I_{g}(t) e^{j\left[\psi_{g}+\phi_{g}(t)\right]}, \quad \text { and } \\
v & =v(t) e^{j\left[\psi_{v}+\phi_{v}(t)\right]} .
\end{aligned}
$$

Substituting the above polar representations into Eq. (6) and equating the real and imaginary parts on both sides of the equality, we have

$\dot{V}+\alpha V=\alpha R\left[I_{g} \cos \left(\phi_{g}-\phi_{V}+\psi_{g}-\psi_{v}\right)-I_{b} \cos \left(\phi_{b}-\phi_{v}-\psi_{v}\right)\right]$,

and

$$
\dot{\phi}_{v}=\Omega-\omega+\frac{\alpha R}{V}\left[I_{g} \sin \left(\phi_{g}-\phi_{v}+\psi_{g}-\psi_{v}\right)-I_{b} \sin \left(\phi_{b}-\phi_{v}-\psi_{v}\right)\right],
$$

where the approximation of $\omega+\Omega \simeq 2 \omega$ has been used.

One can prove that when $\psi_{v}=\psi_{g}$, the system will be in tune; that is, the rf source will see a "real" impedance. In this case, Eqs. (9) and (10) are simplified to 
$\dot{V}+\alpha V=\alpha R\left[I_{g} \cos \left(\phi_{g}-\phi_{V}\right)-I_{b} \cos \left(\phi_{b}-\phi_{V}-\psi_{V}\right)\right]$,

and

$\dot{\phi}_{V}=\Omega-\omega+\frac{\alpha R}{V}\left[I_{g} \sin \left(\phi_{g}-\phi_{v}\right)-I_{b} \sin \left(\phi_{b}-\phi_{v}-\psi_{v}\right)\right]$.

The subsequent analysis will always assume that the system is in tune.

The equations of beam motion, which describe the synchrotron oscillation of proton bunches, can be written as

$\frac{d}{d t}(\Delta E)=\frac{q \omega}{2 \pi h}\left[V \sin \left(\psi_{s}+\phi_{V}-\phi_{b}\right)-V_{s} \sin \psi_{s}\right]$,

and

$\frac{\mathrm{d} \phi_{\mathrm{b}}}{\mathrm{dt}}=\left(\frac{n \omega}{R^{2}}\right)\left(\frac{\Delta E}{E_{0}}\right)$,

where $q$ is the charge of a proton, $v_{s}$ is the cavity voltage in the steady state, $B=\left[1-\left(1 / \gamma^{2}\right)\right]^{1 / 2}, E_{0}$ is the total energy of a synchronous proton, and $\Delta E$ is the energy deviation from $E_{0}$.

Using Eqs. (11)-(14) or equations similar to them, Robinson derived a criterion for the beam-cavity system to be stable. ${ }^{1}$ The criterion written in our notation is

$2 V_{s}\left|\cos \psi_{s}\right|>I_{b} R\left|\sin \left(2 \phi_{y}\right)\right|>0$,

where $\phi_{y}$, the detuning angle of cavities, is defined as

$\phi_{y}=\tan ^{-1}\left(\frac{\Omega-\omega}{\alpha}\right)=\tan ^{-1}\left[\frac{20(\Omega-\omega)}{\Omega}\right]$. 
We note that for stable particle orbits, we need $\Omega \geq \omega$ for $n>0$ and $\Omega \leq w$ for $n<0$.

\section{INJECTION AND EXTRACTION}

For both the booster and the main ring during injection and extraction, the $r f$ field is needed to bunch protons. Therefore the $r f$ frequency should be constant while the resonant frequency of cavities and the phase angles have to satisfy the following conditions:

(i) $\psi_{s}=0, \psi_{g}=\psi_{v}=3 \pi / 2$, and $\Omega>\omega$ for $n>0$,

(ii) $\psi_{s}=\pi, \quad \psi_{g}=\psi_{v}=\pi / 2$, and $\Omega<\omega$ for $\eta<0$.

By requiring $\phi_{b}=\phi_{v}=\dot{\phi}_{v}=0$ in Eqs. (11) and (12), we can

show that

$$
\begin{aligned}
& I_{g}=\left[\left(R I_{b}-\frac{V}{\alpha}|\Omega-w|\right)^{2}+\left(V+\frac{1}{\alpha} \frac{d V}{d t}\right)^{2}\right] 1 / 2 / R \text {, and } \\
& \Phi_{g}=\sin ^{-1}\left\{\left[\left(R I_{b}-\frac{v}{\alpha}|\Omega-\omega|\right) /\left(R I_{g}\right)\right]\left(\frac{\eta}{|n|}\right)\right\} .
\end{aligned}
$$

The Robinson stability criterion can be restated as

$$
\left(\frac{I_{b} R}{V} \cdot\left|\frac{\Omega-\omega}{\alpha}\right|\right)\left|\frac{\Omega-\omega}{\alpha}\right|<1,
$$

where $I_{g}, \phi_{g}, V, I_{b}$, and $\Omega$ are all functions of time.

If $V$ is constant and one wants the minimum $r f$-power consumption, one can take $\phi_{g}=0$ and

$\left|\frac{\Omega-\omega}{\alpha}\right| \approx \frac{I_{b}}{I_{g}}$, 
provided that $\Omega$ can be tuned quickly enough to follow the varying beam current.

In the booster, the beam current will rise and fall linearly during the injection and the slow extraction. If a ramping of total voltage is desired in these periods, then Eqs. (17)-(20) can be used as a guide to programming the rf power and cavity tuning.

The beam-loading problem during the injection into the main ring is a little more complicated than into the booster. First, the step jump of the beam current requires fast tunability of either the $r f$ power or the resonant frequency of cavities. Second, the transient effects caused by the empty buckets, which will occupy one-half the ring, need some kind of servo system to maintain the proper bunching voltage and phase relation. A similar, but more serious, transient beam-loading problem has been solved at Fermilab, where a feedback system has been implemented and is functioning satisfactorily. The same kind of control system could be adopted for LAMPF II.

we now provide a crude estimation of the transient beam-loading effects. From Eq. (2) in the previous section, one can show that the voltage response of the parallel RLC circuit to a step jump of the driving current

$i(t)= \begin{cases}I_{b} e^{j \omega t}, & \text { for } t>0, \\ 0, & \text { for } t \leq 0,\end{cases}$

is roughly

$v(t)=R I_{b} e^{j \omega t}\left(1-e^{-\alpha t} e^{-j \delta \omega t}\right) /\left(1+j \frac{\delta \omega}{\alpha}\right)$,

where $\delta \omega=\omega-\Omega$ and the initial condition of $v(0)=0$ has been assumed. To apply the above equation to the transient beam-loading problem, we take a primitive model in which the empty buckets can be thought of as a pulse of negative current traveling around the ring. A somewhat pessimistic upper bound of the voltage, induced on a cavity because of negative-current pulse, then can be inferred from Eq. (22) as 
$v_{u} \sim R_{b}\left(1-e^{\frac{n \pi t}{Q T}}\right)$

where $\tau$ is the time duration of the negative-current pulse and $T$ is the revolution period of the proton beam.

If we take $R \approx 400 \mathrm{ohm} /$ cavity, $I_{b} \approx 3.2 \mathrm{~A}, Q \approx 1.3 \times 10^{3}, \mathrm{~h}=264$, and $\tau=T / 2$ for a half-filled ring, we find that $V_{u} \approx 45 \mathrm{kV}$. That is almost onehalf of the nominal voltage per cavity proposed. Although the number estimated here may be high, nevertheless it does indicate that a significant voltage drop could occur because of transient beam loading during the injection period of the main ring and shows that a feedback and/or a feedforward control is essential.

In contrast, we have estimated the voltage fluctuation caused by the gap of 12 empty buckets. For this case, $\tau=12 \mathrm{~T} / 264$ and, using Eq. (23), tel1s us that $v_{u} \approx 4 \mathrm{kV}$. This is about $5 \%$ of the nominal voltage per cavity and thus may be tolerated.

\section{ACCELERATION}

The most serious problem in the acceleration stage of LAMPF II is that both the booster and the main ring require substantial rf power. The Robinson stability criterion says that the power dissipated in the cavities should be greater than the power delivered to the beam for the system to be stable. ${ }^{2}$ A design based on this criterion will require an unacceptable amount of $r f$ power for acceleration. An alternative approach is to operate the booster and the main ring beyond the stability limit of the Robinson criterion and to use a feedback scheme similar to the one that has been demonstrated at Fermilab. ${ }^{7}$

Theoretical support for the feedback stabilization of an $r f$ system has been previously documented. ${ }^{3-6}$ In the following, we shall carry out a similar analysis for the parameter range of LAMPF II by using a simplified. feedback model.

To start the analysis, we assume that $I_{b}$ will be a constant and the voltage will have a small error, $\hat{v}$, which is much smaller than the steady-state 
cavity voltage $V_{s}$. We also assume that the generator current $I_{\sim g}$ will contain a steady-state part $\left(I_{g s}, \psi_{g}\right)=\left(I_{g s}, \psi_{v}\right)$ and a small correction, $\left(\zeta, \phi_{g}\right)$ from the feedback circuit; thus,

$$
\begin{aligned}
& v=v_{s}+\hat{v}, \hat{v} \ll v_{s}, \\
& I_{g}=I_{g s}+\zeta, \zeta \ll I_{g s}, \\
& \phi_{g} \ll 1, \\
& I_{b}=\text { constant, and } \\
& I_{g s}=\text { constant. }
\end{aligned}
$$

Note that

$V_{s}=R\left(I_{g s}-I_{b} \cos \psi_{v}\right)$

is the steady-state condition. Substituting the above relations into Eqs. (11)-(14) and linearizing these equations yield

$$
\begin{aligned}
& \frac{d \hat{v}}{d t}+\alpha \hat{v}=\alpha R\left[\zeta-I_{b}\left(\phi_{b}-\phi_{v}\right) \sin \psi_{v}\right], \\
& \frac{d \phi_{v}}{d t}+\alpha \phi_{v}=-\frac{\alpha R \hat{v} I_{b} \sin \psi_{v}}{v_{s}^{2}}+\frac{\alpha R}{V_{s}}\left(I_{g s} \phi_{g}-I_{b} \phi_{b} \cos \psi_{v}\right), \\
& \frac{d}{d t}\left(\frac{\Delta E}{E_{0}}\right)=\frac{a \omega}{2 \pi h E_{0}}\left[V_{s}\left(\phi_{v}-\phi_{b}\right) \cos \psi_{s}+\hat{v} \sin \psi_{s}\right], \text { and } \\
& \frac{d \phi_{b}}{d t}=\left(\frac{n \omega}{\beta^{2}}\right)\left(\frac{\Delta E}{E_{0}}\right) .
\end{aligned}
$$


Making a Laplace transformation with all the initial values set to zero, that is,

$$
\begin{aligned}
& \frac{d \hat{v}}{d t}+s \hat{v}(s), \\
& \frac{d \phi_{v}}{d t}+s \phi_{v}(s), \text { etc., }
\end{aligned}
$$

and using the relations $\sin \psi_{v}=-\cos \psi_{s}, \cos \psi_{v}=\sin \psi_{s}$, and $\tan \phi_{y}=R I_{b} \cos \psi_{s} / V_{s}$, we can derive from Eqs. (26) and (27) the following relations:

$\left(\begin{array}{cc}\alpha+s & \alpha \tan \phi_{y} \\ -\alpha \tan \phi_{y} & \alpha+s\end{array}\right)\left(\begin{array}{c}\hat{v} \\ \\ v_{s} \phi_{v}\end{array}\right)=\alpha R I_{b}\left(\begin{array}{c}\cos \psi_{s} \\ -\sin \psi_{s}\end{array}\right) \phi_{b}+\alpha R\left(\begin{array}{c}\zeta \\ I_{g s} \phi_{a}\end{array}\right)$,

and

$\phi_{g}=\left(\frac{V_{s}}{R I g s}\right)\left[\left(1+\frac{s}{a}\right) \phi_{v}+\tan \phi_{y}\left(\phi_{b} \tan \psi_{s}-\frac{\hat{v}}{V_{s}}\right)\right]$.

Similarly, from Eqs. (28) and (29), we obtain

$$
\phi_{b}=\frac{\Omega_{s}^{2}\left(v_{s} \phi_{v} \cos \psi_{s}+\hat{v} \sin \psi_{s}\right)}{\left(s^{2}+\Omega_{s}^{2}\right) v_{s} \cos \psi_{s}},
$$

where

$$
\Omega_{s}=\left(\frac{q \omega^{2} n V_{s} \cos \psi_{s}}{2 \pi h E_{c} R^{2}}\right)^{1 / 2}
$$


is the steady-state synchrotron frequency.

A lengthy manipulation of Eqs. (31)-(33) yields

$\left(\begin{array}{c}\hat{v} / v_{s} \\ \phi_{g}-\phi_{b}\end{array}\right)=G\left(\begin{array}{l}\zeta^{\prime} / I_{g s} \\ \phi_{g}\end{array}\right)=\left(\begin{array}{l}R I g s \\ V_{s} D\end{array}\right)\left(\begin{array}{ll}d_{11} & d_{12} \\ d_{21} & d_{22}\end{array}\right)\left(\begin{array}{l}\zeta / I g s \\ \phi_{g}\end{array}\right)$,

where

$D=\left(1+\frac{s^{2}}{\Omega_{s}^{2}}\right)\left[\left(1+\frac{s}{\alpha}\right)^{2}+\tan ^{2} \phi_{y}\right]-\frac{I_{b} R \tan \psi_{y}}{V_{s} \cos \psi_{s}}$,

$d_{11}=\left(1+\frac{s}{\alpha}\right)\left(1+\frac{s^{2}}{\Omega_{s}^{2}}\right)+\frac{I_{b} R \sin \psi_{s}}{V_{s}}$,

$d_{12}=-\left(1+\frac{s^{2}}{\Omega_{s}^{2}}\right) \tan \phi_{y}+\frac{I_{b} R \cos \psi_{s}}{V_{s}}$,

$d_{21}=-\left(\frac{V_{s}}{R I_{a s}}\right)\left(1+\frac{I_{b} R \sin \psi_{s}}{V_{s}}\right)\left[\tan \phi_{y}+\left(1+\frac{s}{\alpha}\right) \tan \psi_{s}\right]$, and

$d_{22}=\left(\frac{V_{s}}{R I_{g s}}\right)\left\{\left(\frac{s}{\alpha}\right)\left(1+\frac{s}{\alpha}-\tan \psi_{s} \tan \phi_{y}\right)+\left(\frac{s^{2}}{\Omega_{s}^{2}}\right)\left[\left(1+\frac{s}{\alpha}\right)^{2}+\tan ^{2} \phi_{y}\right]\right\}$.

When there is a small perturbation on the generator current $\left(\zeta, \phi_{g}\right)$,

Eq. (35) relates the cavity voltage deviation $\hat{v}$ and the phase difference between the generator and beam currents, $\phi_{g}-\phi_{b}$, to that perturbation.

A block diagram of the proposed feedback system is shown in Fig. 4. The open-loop transfer matrix for the feedback circuit is

$\left(\begin{array}{c}\zeta / I_{g s} \\ \phi_{g}\end{array}\right)=H\left(\begin{array}{c}\hat{v}_{s} v_{s} \\ \phi_{g}-\phi_{b}\end{array}\right)=\left(\begin{array}{cc}h_{11} & 0 \\ 0 & h_{22}\end{array}\right)\left(\begin{array}{c}\hat{v} / v_{s} \\ \phi_{a}-\phi_{b}\end{array}\right)$, 


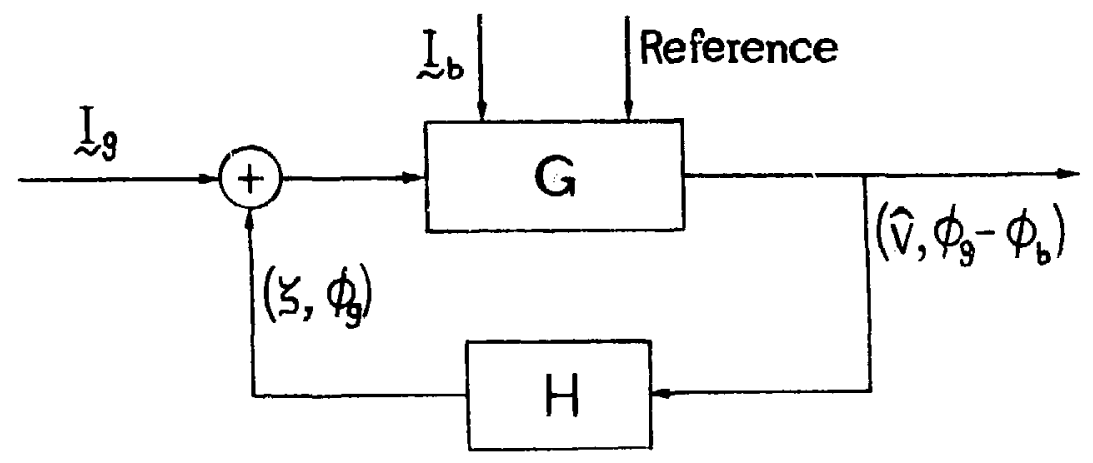

Fig. 4. The proposed rf feedback controls.

where

$$
\begin{aligned}
& h_{11}=A_{v} /\left(1+\frac{s}{P_{v}}\right), \\
& h_{22}=A_{\phi} /\left(1+\frac{s}{P_{\phi}}\right),
\end{aligned}
$$

$A_{v}$ and $A_{\phi}$ are the $d c$ gains, and $P_{v}$ and $P_{\phi}$ are the bandwidths. These singlepole matrix elements correspond to integrating the error signals. The openloop gains of the feedback circuits considered here are characterized by a $20-d B / d e c a d e$ falloff beyond the corner frequencies.

Substituting Eq. (37) into Eq. (35) yields

$$
(\hat{I}-G H)\left(\begin{array}{c}
\hat{v} / V_{s} \\
\phi_{g}-\phi_{b}
\end{array}\right)=0 \text {, }
$$

where $\hat{I}$ is the two-by-two unit matrix. To have a set of nontrivial solutions for these coupled equations, we require that

$$
\operatorname{det}(\hat{I}-G H)=0 .
$$


We therefore have the following characteristic equation:

$$
\begin{gathered}
\left(1+\frac{s}{P_{v}}\right)\left(1+\frac{s}{P_{\phi}}\right) D-\left(\frac{R I_{g s}}{v_{s}}\right)\left[A_{v}\left(1+\frac{s}{P_{\phi}}\right) d 1+A_{\phi}\left(1+\frac{s}{P_{v}}\right) d_{22} j\right. \\
+\left(\frac{R I_{q s}}{v_{s}}\right) A_{v} A_{\phi}\left[\left(1+\frac{s^{2}}{\Omega_{s}^{2}}\right)\left(1+\frac{s}{\alpha}\right)-1\right]=0 .
\end{gathered}
$$

This is a six-order algebraic equation. For the system to be stable, all six roots of Eq. (42) must have negative real parts. Thus, to achieve the feedback stabilizations, we need to find the values of $A_{v}, A_{\phi}, P_{v}$, and $P_{\phi}$ so that all solutions to the above equation will have negative real parts. It seems there is no simple way to do it. One could start with some assumed initial values for the dc gains and bandwidths, then vary these parameters and inspect the roots of $\mathrm{Eq}$. (42) to locate a reasonable working region in the parameter space.

As an example, we shall consider a possible design for the booster of LAMPF II. The values of the relevant parameters are listed in Table I. Because the rf parameters of the main ring and the booster are in the same range and because the purpose of the current study is to provide an estimation only, we shall not consider the main ring here.

\section{TABLE I}

\section{POSSIBLE $r f$ PARAMETERS FOR THE LAMPF II BOOSTER}

Twice the average beam current per pulse Synchronous angle

Cavity detuning angle

Maximal voltage per cavity

Shunt resistance per cavity

$1 /$ (cavity time constant)

Steady-state synchrotron frequency

Steady-state synchrotron tune

Power ratio

$\begin{array}{ll}\mathrm{I}_{\mathrm{b}} & 3.2 \mathrm{~A} \\ \psi_{\mathrm{s}} & 45^{\circ} \\ \phi_{\mathrm{y}} & 50^{\circ} \\ \mathrm{V}_{\mathrm{s}} & 90 \mathrm{kV} \\ \mathrm{R} & 47.4 \mathrm{k} \Omega \\ \alpha & 1.66 \times 10^{5} / \mathrm{s} \\ \Omega_{\mathrm{S}} & 3.14 \times 10^{4} / \mathrm{s} \\ \nu_{\mathrm{S}} & 0.01 \\ \mathrm{P}_{\mathrm{R}} & 1.2\end{array}$


Without the feedback, Eq. (42) reduces to $D=0$, which has four roots. For the numbers tabulated in Table I, the power ratio is larger than unity; therefore, according to the Robinson stability criterion, the system should be unstable. Soiving the equation of $D=0$, we find that the maximal growth rate is about $0.34 \Omega_{\mathrm{s}}$, which corresponds to an e-folding time of 47 turns.

If we insert a feedback circuit as indicated in Fig. 4 and Eqs. (37)-(39) with $A_{v}=-500, A_{\phi}=-4, P_{v}=0.1 \Omega_{s}$, and $P_{\phi}=0.5 \Omega_{s}$, we find that the system is stabilized with a minimal damping rate of $0.6 \Omega \mathrm{s}$, which corresponds to an efolding time of 27 turns. The loci of the three roots with $\operatorname{Re}(s)>-\Omega_{S}$ are shown in Figs. 5-8 as functions of $A_{v}, A_{\phi}, P_{v}$, and $P_{\phi}$, respectively. In $e$ ach figure, only one of these four feedback circuit parameters is varied. Three other roots, not shown in the figures, have real parts less than the steadystate synchrotron frequency. Those modes correspond to strongly damped oscillations even in the absence of the feedback. We note again that the feedback circuit parameters cited above do not represent the best case. The damping rate can be improved by suitably tailoring the feedback circuit and carefully examining the parameters. Nonetheless, the above-mentioned parameter values show that the use of feedback control to stabilize the rf system and to achieve a greater-than-one power ratio in LAMPF II should not present any challenge to existing technology. 


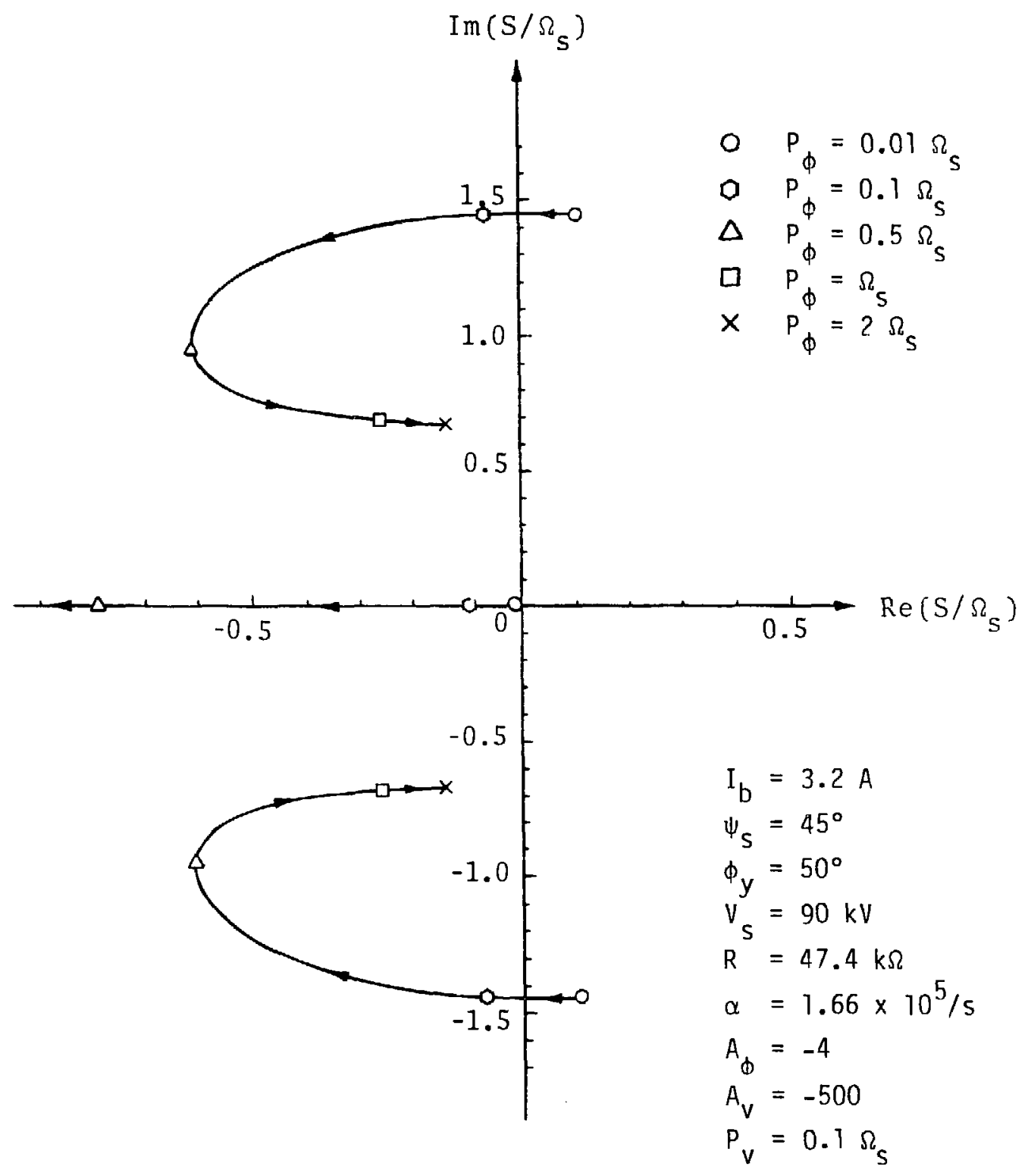

Fig. 5. Loci of three out of the six roots of $E q .(42)$, with $\operatorname{Re}(s)>-\Omega_{s}$, as functions of $P_{\phi}$. Relevant parameter values are shown in the diagram. 


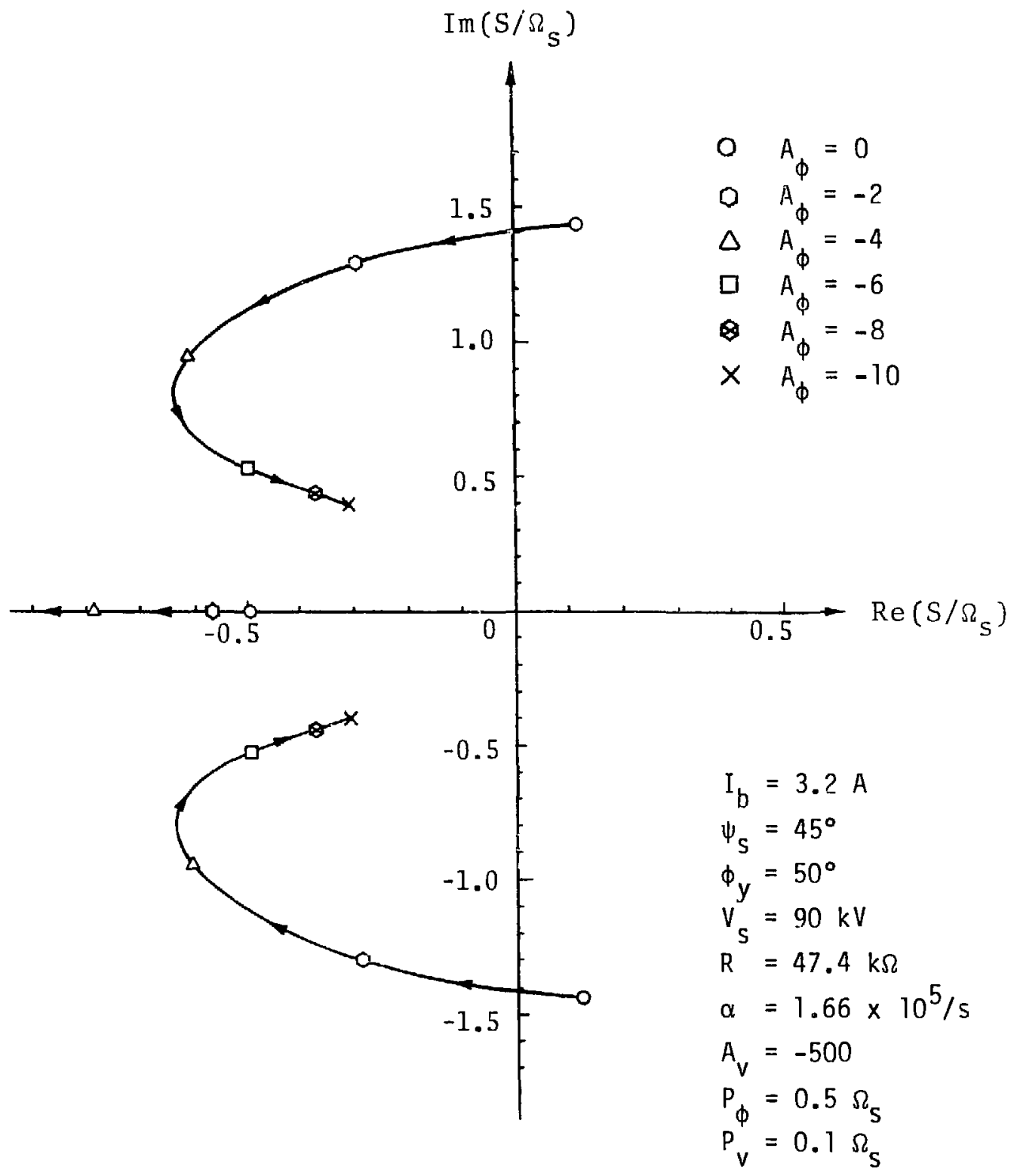

Fig. 6. Loci of three out of the six roots of $E q .(42)$, with $\operatorname{Re}(s)>-\Omega_{s}$, as functions of $A_{\phi}$. Relevant parameter values are shown in the diagram. 


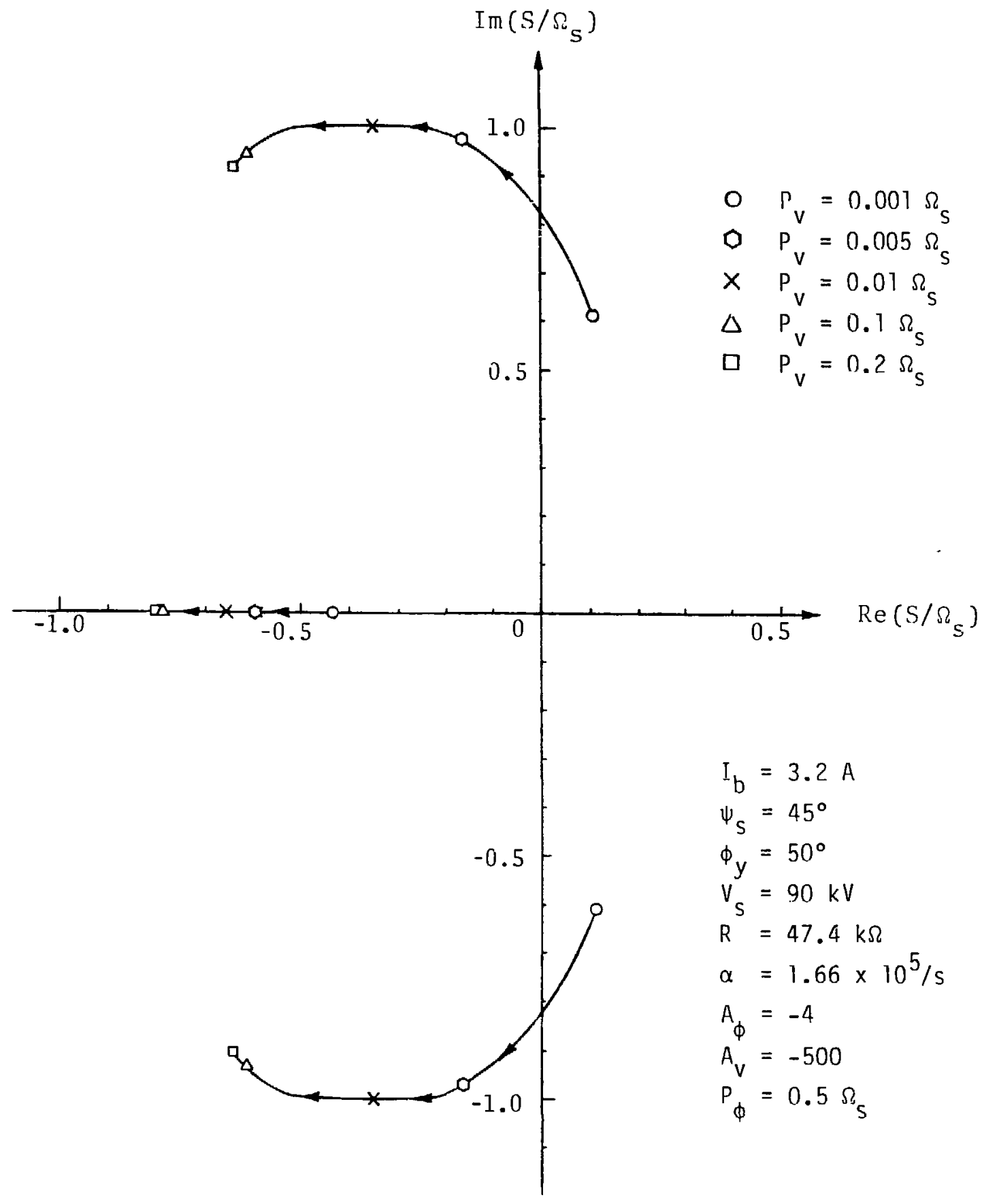

Fig. 7. Loci of three out of the six roots of Eq. (42), with $\operatorname{Re}(s)>-\Omega_{s}$, as functions of $P_{v}$. Relevant parameter values are shown in the diagram. 


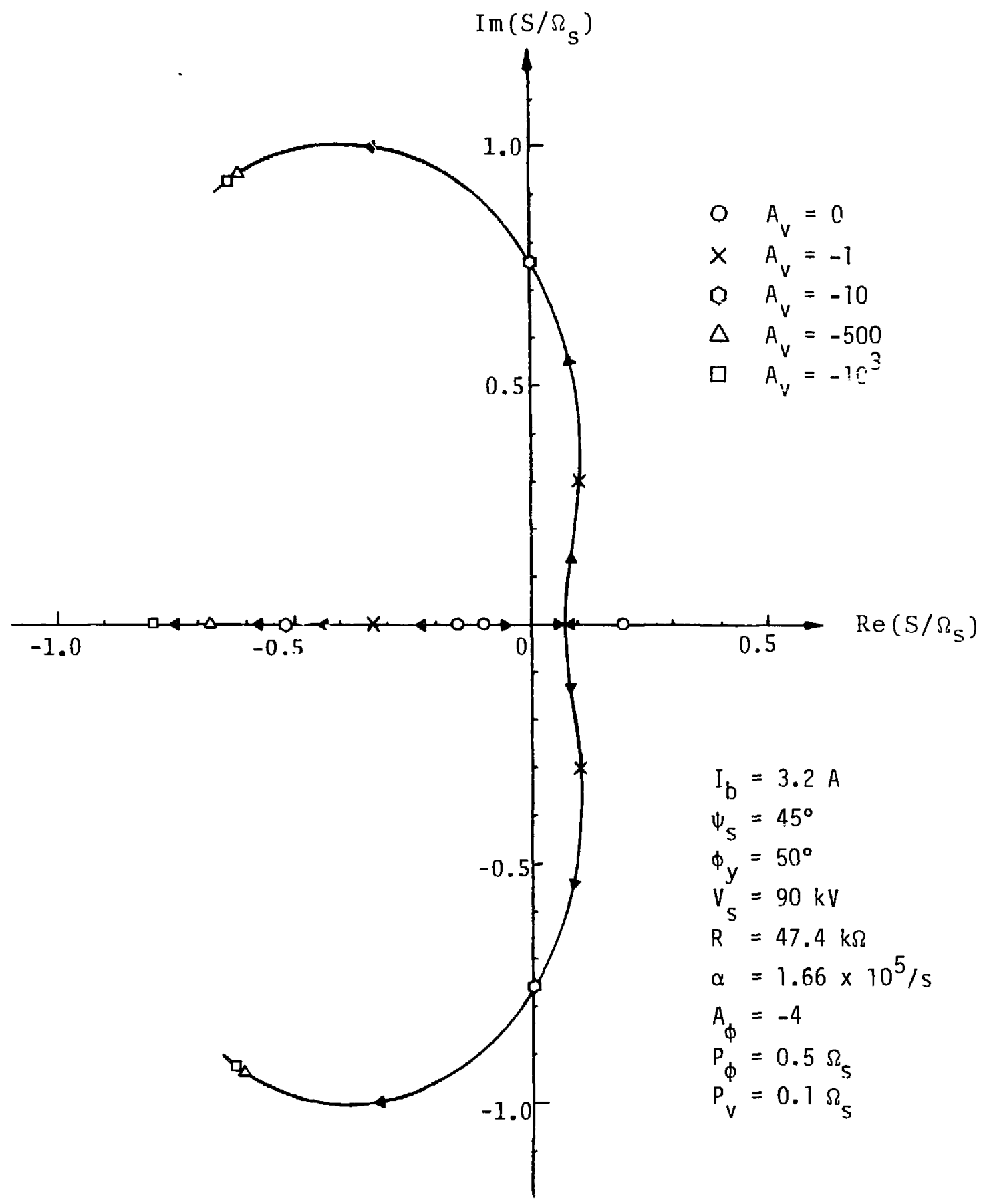

Fig. 8. Loci of three out of the six roots of Eq. (42), with $\operatorname{Re}(s)>-\Omega_{s}$, as functions of $A_{v}$. Relevant parameter values are shown in the diagram. 


\section{CONCLUSIONS}

We have pointed out some of the beam-ioading problems for LAMPF II. The important ones are the transi effects in the injection period of the main ring arid the power consumption in the acceleration stage. Both of these problems can be siived by implementing proper feedforward and feedback controls, according to James Griffin of Ferinilab.

An estimation of the feedback control requirements for achieving the acceleration of protons in the range of power ratio greater than une is provided. The anaiysis is based on the equivalent circuit model for the beam-casity system. The feedback control study is limited to the linear regime. For simplicity, we have neglected the variation of revolution frequency caused by the acceleration. Also, we have not attempted to optimize the feedback design in this study. The results of this simple estimation show that there will be no technical difficulty in reaching the goal of a power ratio greater than one in LAMPF II.

\section{ACKNOWLEDGMENT}

The authors would like to thank James Griffin and Edward Hiqqins for their helpful discussion.

\section{REFERENCES}

1. K. W. Robinson, "Stability of Beam in Radio-Frequency System," Cambridge Electron Accelerator internal report No. CEAL-1010 (1964).

2. J. E. Griffin, "A Numerical Example of an RF Accelerating System," in "Physics of High Energy Particle Accelerators," Proc. of Fermilab Summer School, 1981, p. 564, (1982).

3. M. Donald, E. A. Hughes and D. J. Thompson, "RF Cavity Voltage Stabilizing System," Daresbury internal report EL/TM/37 (1966).

4. M. Lee, "Beam-RF Cavity Stability with Feedback Control in a Circular Accelerator," IEEE Trans. Nuc1. Sci., NS-18 (3) 1086 (1971).

5. F. Pedersen, "Beam Loading Effects in the CERN PS Booster," IEEE Trans. Nuc 1. Sci., NS-22 (3) 1906 (1975). 
6. D. J. Bell, P. A. Cook, N. Munro and T. E. Swain, "Some Considerations of Feedback Loop Design for the SRS Storage Ring RF System," IEEE Trans. Nucl. Sci., NS-29 (5) 1415 (1982).

7. J. E. Griffin, "Compensation for Beam Loading in the 400-GeV Fermilab Main Accelerator," IEEE Trans. Nucl. Sci., NS-2i (3) 1910 (1975). 